ATOMIC DATA AND NUCLEAR DATA TABLES 17, 467-473 (1976)

\title{
MASSES FROM INHOMOGENEOUS PARTIAL DIFFERENCE EQUATIONS*
}

\author{
J. JÄNECKE and B. P. EYNON ${ }^{\dagger}$ \\ Department of Physics, University of Michigan \\ Ann Arbor, Michigan 48109
}

\begin{abstract}
Procedures are described for obtaining mass predictions from the solutions of inhomogeneous partial difference equations. The inhomogeneous contributions result from the variation with nucleon number and neutron excess of the effective neutron-proton interaction. A simple liquid-drop-model expression has been used for these contributions to obtain the present predictions. The most general solutions of the difference equation have been subjected to a $\chi^{2}$-minimization procedure (boundary condition) based on the new atomic mass adjustment of Wapstra and Bos. The resulting solution can be viewed as a many-parameter mass equation with about 220 parameters. About 5000 mass values have been calculated for nuclei with $A \geq 65$. The standard deviation between calculated and experimental mass-excess valucs is $\sigma_{m}=289 \mathrm{keV}$.
\end{abstract}

\footnotetext{
* Supported in part by the U. S. Energy and Research Development Administration Contract E(11-1)-2167 and by a research grant from the Michigan Memorial Phoenix Project, University of Michigan

$\dagger$ Now at the Department of Statistics, Stanford University, Stanford, California 94305
}

Copyright (C) 1976 by Academic Press, Inc. 


\section{CONTENTS}

\section{INHOMOGENEOUS PARTIAL DIFFERENCE EQUATIONS}

\section{CALCULATIONS AND COEFFICIENTS}

\section{INHOMOGENEOUS PARTIAL DIFFERENCE EQUATIONS}

Many mass equations $M(N, Z)$ are obtained as analytic expressions from nuclear-structure considerations. While the underlying theory or model, such as the liquid-drop model or the shell model, establishes the analytic form of the equations, some of the parameters contained in the equations are generally not, or only poorly, predicted by the theory. These parameters are subsequently determined by minimizing the differences $M(N, Z)-M_{\text {exp }}(N, Z)$ for all known masses.

A different approach by means of inhomogeneous partial difference equations is described in the present contribution (see Refs. 1-3 for details). If $M_{\text {exact }}(N, Z)$ represents the exact masses of all known and unknown nuclei, the objective is to find a mass equation $M(N, Z)$ which satisfies

$$
M(N, Z)=M_{\text {exact }}(N, Z)
$$

[or more realistically, of course, $M(N, Z) \approx M_{\text {exact }}(N, Z)$ ]. If $D$ represents a partial difference operator, then

$$
D M(N, Z)=[D M(N, Z)]_{\text {exact }}
$$

is also correct. We now invert the problem. If $[D M(N, Z)]_{\text {exact }}$ is assumed to be known from nuclearstructure theories and if the inhomogeneous partial difference Eq. (2) has a unique solution $M(N, Z)$, then $M(N, Z)=M_{\text {exact }}(N, Z)$. In order to obtain a unique solution over a range of $N$ and $Z$ values, it must be required (boundary condition) to reproduce all precisely known experimental masses $M_{\text {exp }}(N, Z)$. It is concluded that very limited information about $M_{\text {exact }}(N, Z)$ may be sufficient to derive an exact mass equation.

Solutions $M(N, Z)$ of the inhomogeneous partial difference equation

$$
D M(N, Z)=[D M(N, Z)]_{\text {theor }}
$$

based on approximate theories or assumptions for

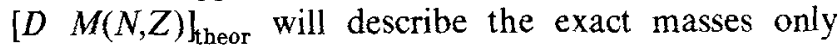
approximately, $M(N, Z) \approx M_{\text {exact }}(N, Z)$. The earlier boundary condition has to be replaced by a $\chi^{2}$-minimization of the differences $M(N, Z)-M_{\text {exp }}(N, Z)$, and the range of validity is that for which Eq. (3) yields unique solutions.

Furthermore, if two independent operators $D_{T}$ and $D_{L}$ can be found with theoretical predictions
$\left[D_{T} M(N, Z)\right]_{\text {theor }}$ and $\left[D_{L} M(N, Z)\right]_{\text {theor }}$, then the resulting solutions $M_{T}(N, Z)$ and $M_{L}(N, Z)$ of the inhomogeneous partial difference equations

$$
D_{T} M(N, Z)=\left[D_{T} M(N, Z)\right]_{\text {theor }}
$$

and

$$
D_{L} M(N, Z)=\left[D_{L} M(N, Z)\right]_{\text {thcor }}
$$

must satisfy $M_{L}(N, Z)-M_{T}(N, Z) \approx 0$ for all values of $N$ and $\mathcal{Z}$ for which both solutions are unique. This is a necessary condition, and the degree to which it is violated, particularly for neutron-rich and proton-rich nuclei, makes it possible ${ }^{3}$ to judge the reliability of the underlying theoretical assumptions and with it the reliability of mass predictions.

Another possible approach ${ }^{2}$ for treating the two Eqs. (4) and (5) consists of finding those solutions which satisfy both difference equations simultaneously with the original boundary condition again replaced by the $\chi^{2}$-minimization.

The theoretical contributions on the right-hand side of Eqs. (4) and (5) generally contain small errors. The solutions $M_{T}(N, Z)$ and $M_{L}(N, Z)$ may therefore include systematic errors which will become important for neutron-rich and proton-rich nuclei. Such systematic errors can be reduced ${ }^{2}$ if the solutions are subjected to constraints which, for example, ensure that the solutions satisfy charge symmetry of nuclear forces or ensure reasonable predictions for the Coulomb energies.

Partial difference operators $D_{T}$ and $D_{L}$ have been constructed from operators ${ }^{m, n} \Delta$ defined by

$$
{ }^{m, n} \Delta f(N, Z) \equiv f(N, Z)-f(N-m, Z-n)
$$

(this definition differs slightly from that used in Ref. 3). The quantity

$$
I_{n p}(N, Z) \equiv{ }^{1,0} \Delta^{0,1} \Delta B(N, Z)=-{ }^{1,0} \Delta^{0,1} \Delta M(N, Z)
$$

$[B(N, Z)=$ binding energy $]$ represents essentially the effective neutron-proton interaction ${ }^{1-4} I_{n p}$. The effective interaction $I_{n p}$ is responsible for the symmetry energy term in mass equations. Additional small contributions to $I_{n p}$ result from the neutron-proton pairing energy, from the Coulomb energy (since the isotope-shift coefficient of the nuclear-charge radius is generally different from zero), and from collective effects. Partial difference equations in accord with the above general considera- 
tions can now be obtained by considering the dependence on the neutron excess $N-Z=2 T_{Z}$ and the nucleon number $N+Z=A$ of the quantity $I_{n p}$. We therefore define the transverse and longitudinal partial difference operators

$$
D_{T} \equiv-{ }^{1,-1} \Delta^{1,0} \Delta^{0,1} \Delta
$$

and

$$
D_{L} \equiv-{ }^{1,1} \Delta^{1,0} \Delta^{0,1} \Delta
$$

For the contributions which vary smoothly with $N$ and $Z$ (or $A$ and $T_{Z}$ ), Eqs. (7), (8), and (9) can be written approximately as

$$
\begin{aligned}
I_{n p}(N, Z) \approx \frac{\partial^{2}}{\partial N \partial Z} M(N, Z) & \\
= & -\left(\frac{\partial^{2}}{\partial A^{2}}-\frac{1}{4} \frac{\partial^{2}}{\partial T_{Z}^{2}}\right) M(N, Z), \\
D_{T} \approx-\left(\frac{\partial}{\partial N}-\frac{\partial}{\partial Z}\right) & \frac{\partial^{2}}{\partial N \partial Z} \\
& =-\frac{\partial}{\partial T_{Z}}\left(\frac{\partial^{2}}{\partial A^{2}}-\frac{1}{4} \frac{\partial^{2}}{\partial T_{Z}^{2}}\right), \\
D_{L} \approx-\left(\frac{\partial}{\partial N}+\frac{\partial}{\partial Z}\right) & \frac{\partial^{2}}{\partial N \partial Z} \\
& =-2 \frac{\partial}{\partial A}\left(\frac{\partial^{2}}{\partial A}-\frac{1}{4} \frac{\partial^{2}}{\partial T_{Z}^{2}}\right) .
\end{aligned}
$$

The inhomogeneous partial difference Eqs. (4) and (5) based on the operators of Eqs. (8) and (9) are sche-
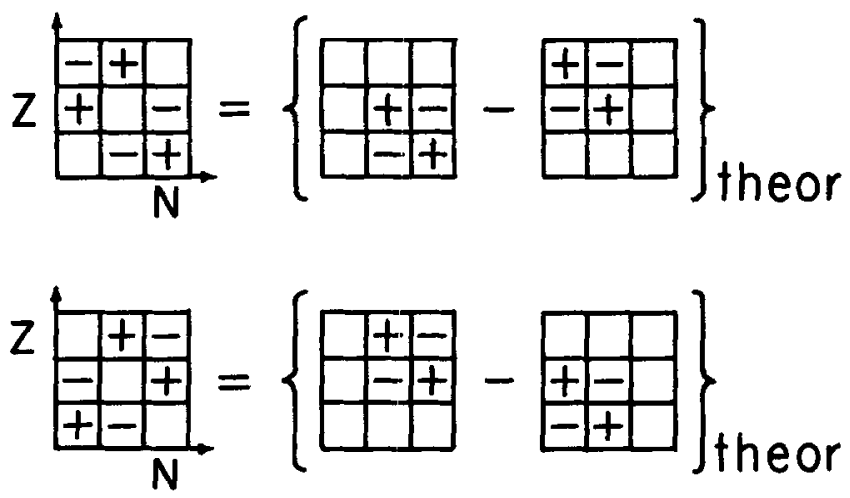

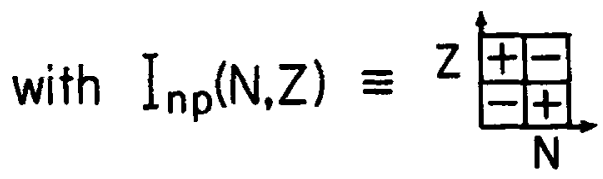

Fig. 1. Schematic representation of the transverse and longitudinal inhomogeneous partial difference Eqs. (4) and (5) based on the operators $D_{T}$ and $D_{L}$ of Eqs. (8) and (9). The boxes represent nuclei from the nuclidic chart with $N$ horizontal and $Z$ vertical. The presence of a plus or a minus sign in a box indicates that the mass value of the respective nucleus is to be added or subtracted matically represented in Fig. 1. Included in the figure is the schematic representation of the definition for the effective neutron-proton interaction $I_{n p}$ from Eq. (7). The connection with the Garvey-Kelson nuclidic mass relations ${ }^{5}$ becomes quite apparent in this notation. The transverse and longitudinal Garvey-Kelson relations are represented by the homogeneous partial difference equations

and

$$
D_{T} M(N, Z)=0
$$

$$
D_{L} M(N, Z)=0 .
$$

Figure 2 shows about 500 values for $I_{n p}$ calculated from Eq. (7) and the experimental masses of Ref. 6. The data are plotted as a function of $A$ separately for even- $A$ and odd- $A$ nuclei. The even- $A$-odd- $A$ effect has been explained by de-Shalit ${ }^{4}$ who showed that $I_{n p}$ can be written in the form

$$
I_{n p}=I_{0}+(-1)^{A} I^{\prime} .
$$

Here, $I_{0}$ represents an averaged interaction between a

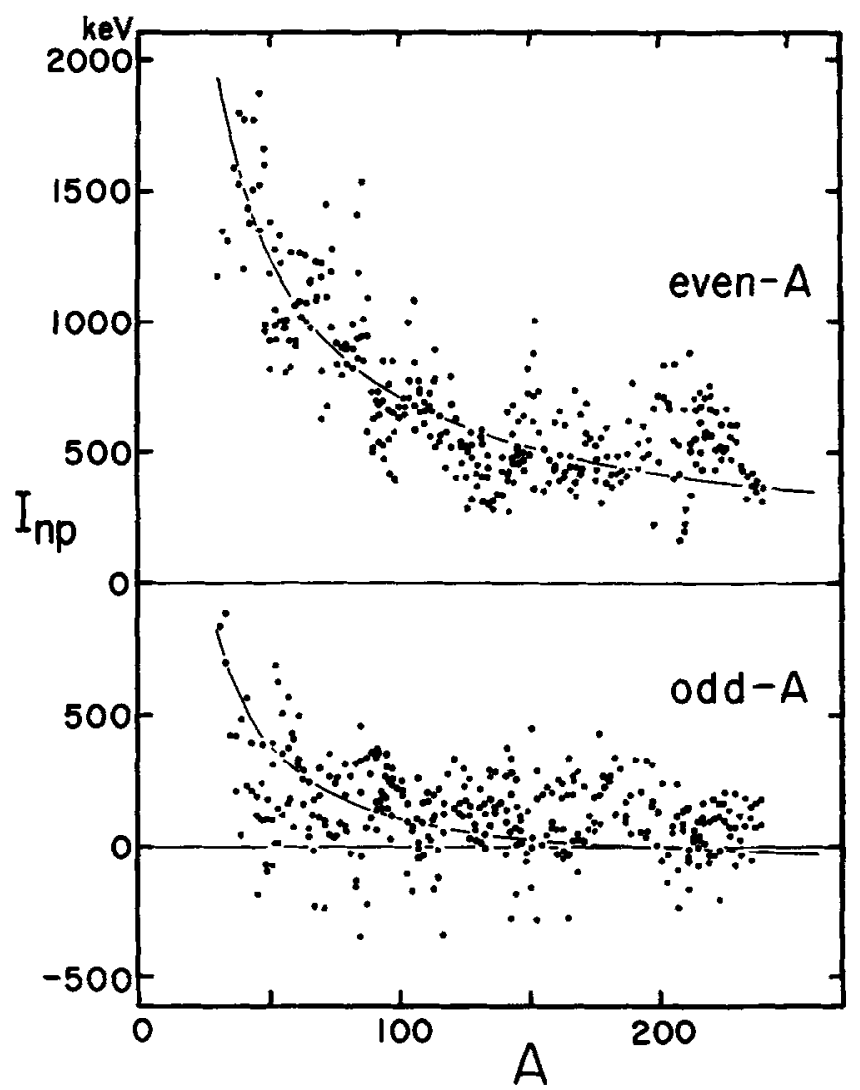

Fig. 2. Plot of the effective neutron-proton interaction $I_{n p}$ derived from the experimental masses as a function of $A$ by means of Eq. (7). The lines are calculated from the Bethe-Weizsäcker liquiddrop-model equation for nuclei along the line of $\beta$-stability (figure taken from the first article in Ref. 1; B-W parameters used are listed there) 
neutron and a proton in the outermost shells of an odd-odd nucleus while $I^{\prime}$ accounts for the increased binding (pairing energy) in the ground state. The overall behavior is quite well described even by simple mass equations. The two lines in Fig. 2 are calculated ${ }^{1}$ for nuclei along the line of $\beta$-stability from the BetheWeizsäcker liquid-drop-model mass equation. The experimental evidence for the dependence on the two variables nucleon number and neutron excess is discussed in Ref. 1 .

General solutions of the inhomogeneous thirdorder transverse and longitudinal partial difference equations

$$
-{ }^{1,-1} \Delta^{1,0} \Delta^{0,1} \Delta M(N, Z)=\left[{ }^{1,-1} \Delta I_{n p}(N, Z)\right]_{\text {theor }}
$$

and

$$
-{ }^{1,1} \Delta^{1,0} \Delta^{0,1} \Delta M(N, Z)=\left[1,1 \Delta I_{n p}(N, Z)\right]_{\text {theor }}
$$

[Eqs. (4) and (5) with (8) and (9)] consist of a particular solution of the inhomogeneous equation and the most general solution of the homogeneous equation. Solutions can easily be obtained if certain simple assumptions are made about the $A$ - and $T_{Z}$-dependence of $I_{n p}(N, Z)=I_{n p}\left(A, T_{Z}\right)$ which convert Eqs. (16) and (17) into homogeneous equations. If $I_{n p}$ is assumed to be independent of $T_{Z}$ or independent of $A$, then

$$
M(N, Z)=g_{1}(N)+g_{2}(Z)+g_{3}(N+Z)
$$

and

$$
M(N, Z)=f_{1}(N)+f_{2}(Z)+f_{3}(N-Z)
$$

are the most general solutions of the homogeneous transverse and longitudinal Eqs. (16) and (17), respectively. If $I_{n p}$ is assumed to be independent of $T_{z}$ and $A$ (separately for even- $A$ and odd- $A$ ), then Eqs. (18) and (19) represent again the most general solutions, and the most general simultaneous solution is

$$
\begin{aligned}
M(N, Z)=h_{1}(N) & +h_{2}(Z)+\eta_{1}(N-Z)^{2} \\
& +\eta_{2} \delta_{o o}+\eta_{3} \delta_{e e}+\eta_{4} \delta_{e o}+\eta_{5} \delta_{o e} .
\end{aligned}
$$

Here, $g_{i}(k), f_{i}(k)$, and $h_{i}(k)$ are arbitrary functions, and the $\eta_{i}$ are arbitrary constants. The quantity $\delta_{00}$ is unity for $N=$ odd, $Z=$ odd and is zero otherwise. The quantities $\delta_{e e}, \delta_{e o}$ and $\delta_{o e}$ have similar meanings. The functions $h_{1}(N)$ and $h_{2}(Z)$ must contain nuclear and Coulomb energy contributions to satisfy charge symmetry of nuclear forces. Thus,

$$
h_{1}(N)+h_{2}(Z)=h_{\text {nucl }}(N)+h_{\text {nucl }}(Z)+h_{\text {Coul }}(Z)
$$

with

$$
\begin{aligned}
& h_{\text {nucl }}(k)=h_{1}(k), \\
& h_{\text {Coul }}(k)=h_{2}(k)-h_{1}(k) .
\end{aligned}
$$

The same is the case for the functions $g_{i}(k)$ and $f_{i}(k)$ as well as for the functions $G_{i}(k), F_{i}(k)$, and $H_{i}(k)$ introduced below. The functions $g_{i}(k), f_{i}(k)$, and $h_{i}(k)$ can be determined from a $\chi^{2}$-minimization to the experimentally known masses. Equations (18) and (19) represent, of course, the transverse and longitudinal Garvey-Kelson mass equations. ${ }^{5}$ Since the above assumptions are strongly violated near $T=0$, the solutions can be used only for nuclei with $N>Z$ (and $N=Z=$ even).

Many theoretical expressions for the right-hand sides of the inhomogeneous partial difference Eqs. (16) and (17) are easily available. Any given mass equation $M_{\text {eq }}(N, Z)$ contains terms which describe the dependence on $T_{Z}$ and $A$ of the effective interaction $I_{n p}$. These terms, mostly contained in the expression for the symmetry energy, can be obtained by calculating the required differences. The underlying theoretical considerations for these contributions are, of course, those used in the derivation of the respective mass equation. The most general solutions of Eqs. (16) and (17) then become

$$
\begin{aligned}
& M(N, Z) \\
& \quad=M_{\text {eq }}(N, Z)+G_{1}(N)+G_{2}(Z)+G_{3}(N+Z)
\end{aligned}
$$

and

$$
\begin{aligned}
& M(N, Z) \\
& \quad=M_{\mathrm{eq}}(N, Z)+F_{1}(N)+F_{2}(Z)+F_{3}(N-Z) .
\end{aligned}
$$

The most general simultaneous solution is

$M(N, Z)$

$$
\begin{aligned}
=M_{e q}(N, Z) & +H_{1}(N)+H_{2}(Z)+\eta_{1}(N-Z)^{2} \\
& +\eta_{2} \delta_{o o}+\eta_{3} \delta_{e e}+\eta_{4} \delta_{e o}+\eta_{5} \delta_{o e} .
\end{aligned}
$$

Here, $G_{i}(k), F_{i}(k)$, and $H_{i}(k)$ are arbitrary functions and the $\eta_{i}$ are arbitrary constants. These can again be determined from a $\chi^{2}$-minimization of the differences between experimental and calculated masses for those regions of $N, Z, N+Z$, and $N-Z$ for which experimental masses are known. While the expression for $M_{\text {eq }}(N, Z)$ enters explicitly into the solutions (23), (24), and (25), the only quantities which these solutions and $M_{\text {eq }}(N, Z)$ have in common are certain third-order partial differences which are generally on the order of $10 \mathrm{keV}$ (see Fig. 5 of Ref. 1).

\section{CALCULATIONS AND COEFFICIENTS}

Computer programs have been written by us which make it possible to obtain the functions $G_{i}(k)$, $F_{i}(k)$, and $H_{i}(k)$ as numerical values for each integer argument from systems of a few hundred linear equations in a few hundred unknowns. Since use is made of sparse matrix subroutines, the computing time is only about ten seconds for a given equation on the University 
of Michigan AMDAHL 470V/6 computer and about twice as long on the compatible IBM $370 / 168$.

Preliminary results for the transverse and longitudinal inhomogeneous equations according to Eqs. (23) and (24) have been obtained ${ }^{3}$ for several shell-model and liquid-drop-model expressions for $I_{n p}$. The standard deviations $\sigma_{m}$ between calculated and experimental mass-excess values are typically $110 \mathrm{keV}$ and $200 \mathrm{keV}$, respectively. The consistency test mentioned earlier has been applied to a few cases. ${ }^{3}$ Detailed results are available for the simultaneous solutions according to Eq. (25). Solutions have been obtained ${ }^{2}$ for 16 different assumptions and theories about $I_{n p}(N, Z)$ including shellmodel $^{7,8}$ and liquid-drop-model ${ }^{9-11}$ expressions. The standard deviations $\sigma_{m}$ between calculated and experimental mass-excess values $(N \geq 20$ and $Z \geq 20)$ are typically $250 \mathrm{keV}$. However, it was found that the standard deviations $\sigma_{c}$ for reproducing the experimental Coulomb displacement energies were much bigger and ranged from about 650 to $1750 \mathrm{keV}$. The functions $H_{\text {nucl }}(k)$ and $H_{\text {Coul }}(k)$ display a divergent behavior. It was further observed that there exist strong correlations between the Coulomb energy and the symmetry energy terms. A misrepresentation of the former is always accompanied by a misrepresentation of the latter thus affecting mass predictions for very neutron-rich and proton-rich nuclei.

A similar situation exists for the transverse Garvey-Kelson mass-equation. ${ }^{5}$ The standard deviation $\sigma_{m}$ for the differences between calculated and experimental mass-excess values is about $120 \mathrm{keV}$, but the calculated Coulomb displacement energies exhibit deviations with $\sigma_{c} \approx 2700 \mathrm{keV}$.

Additional constraints were introduced in order to overcome this problem. Since the terms $H_{1}(N)$ and $H_{2}(Z)$ of Eq. (25) contain nuclear and Coulomb energy contributions, obvious constraints are $H_{1}(k)=H_{2}(k)$ or $\eta_{1}=0$ which eliminate any modifications of the Coulomb energy or symmetry energy terms of $M_{\mathrm{eq}}(N, Z)$. Results were again obtained from $\chi^{2}$-minimizations for the various assumptions and theories about $I_{n p}$. The functions $H_{\text {nucl }}(k) \equiv H_{1}(k)$ and $H_{\text {Coul }}(k) \equiv H_{2}(k)-H_{1}(k)$ are now well behaved. Figure 3 shows an example based on the constraint $\eta_{1}=0$. The dependence of $H_{\text {Coul }}(Z)$ on $Z$ is smooth, and $H_{\text {nucl }}(N$ or $Z)$ displays pronounced shell and pairing effects as expected.

Of the many new mass equations obtained, one was chosen for presentation in this contribution (referred to as solution S-C in Ref. 2). It is the simultaneous solution Eq. (25) of the inhomogeneous equations derived under the constraint $\eta_{1}=0$ with the effective neutron-proton interaction $I_{n p}$ taken from the liquiddrop-model expression of Seeger. ${ }^{11}$ It should be used only for $A \geq 65$. The dependence of the effective neu- tron-proton interaction $I_{n p}$ on shell-model configurations becomes too important in light nuclei and cannot be neglected. The selection of the above solution is based on the standard deviations $\sigma_{m}$ and $\sigma_{c}$ in conjunction with their variation under constraints (see Ref. 2 for more details).

The functions $H_{1}(N)$ and $H_{2}(Z)$ were obtained from a slightly modified $\chi^{2}$-minimization procedure by solving a system of about 220 linear equations in about 220 unknowns. The new experimental mass values of Wapstra and Bos ${ }^{12}$ were used as input data. By quadratically adding $100 \mathrm{keV}$ to the experimental uncertainties, values with uncertainties less than $100 \mathrm{keV}$ are thereby given essentially equal weight, and reduced weight is given to those with larger uncertainties.

Mass excesses $\Delta M(N, Z)$ are calculated from

$\Delta M(N, Z)$

$$
\begin{aligned}
=\Delta M_{e q}(N, Z) & +H_{1}(N)+H_{2}(Z)+\eta_{1}(N-Z)^{2} \\
& +\eta_{2} \delta_{o o}+\eta_{3} \delta_{e e}+\eta_{4} \delta_{e o}+\eta_{5} \delta_{o e}
\end{aligned}
$$

(the $\delta_{o o}$ etc. are again Kronecker symbols) with

$$
\begin{aligned}
\Delta M_{\mathrm{eq}}(N, Z)= & N \Delta M_{n}+Z \Delta M_{H}-B_{\mathrm{eq}}(N, Z), \quad(27) \\
B_{\mathrm{eq}}(N, Z)= & \alpha A-\beta \frac{(N-Z)^{2}}{A}-\gamma A^{2 / 3}+\eta \frac{(N-Z)^{2}}{A^{4 / 3}} \\
& -864 \frac{Z^{2}}{r_{0} A^{1 / 3}}\left\{1-\frac{0.76361}{Z^{2 / 3}}-\frac{2.453}{r_{0}{ }^{2} A^{2 / 3}}\right\} \\
& +7000 \exp \left(-6 \frac{|N-Z|}{A}\right) \\
& +14.33 \times 10^{-3} Z^{2.39} .
\end{aligned}
$$

With the exception of the pairing energy term $\pm(9600 \mathrm{keV}) A^{-1 / 2}$ which is replaced by contributions to

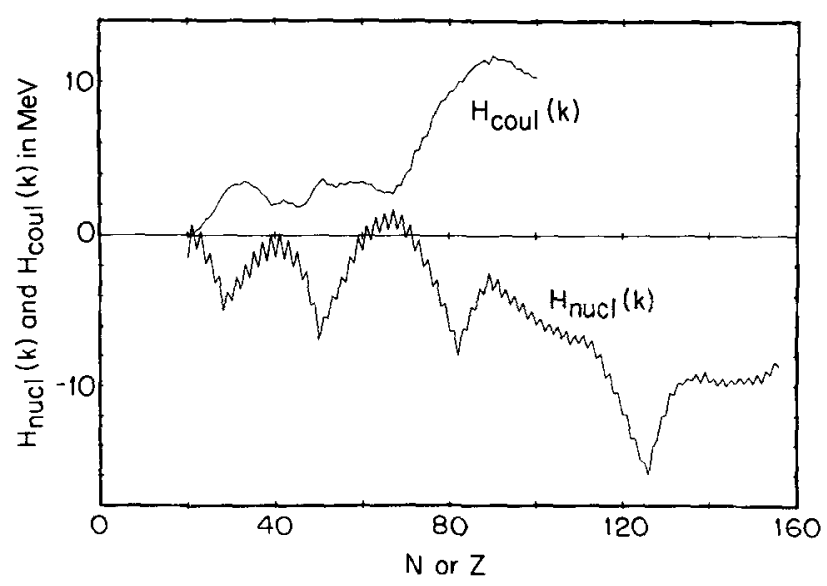

Fig. 3. Plot of the functions $H_{\text {uucl }}(k) \equiv H_{1}(k)$ and $H_{\mathrm{coul}}(k) \equiv H_{2}(k)$ - $H_{1}(k)$ for the simultaneous solution, Eq. (26) with Eqs. (27) and (28). of the inhomogeneous partial difference Eqs. (4) and (5) 
$H_{1}(N)$ and $H_{2}(Z)$. Eq. (28) is a hinding energy expression of Seeger. ${ }^{11}$ The small third-order differences in the inhomogeneous partial difference Fqs. (16) and (17) depend essentially only on the symmetry energy terms with $\beta$ and $\eta$. All quantities are in units of $\mathrm{keV}$.

\section{Values of Coefficients}

$\begin{array}{rlrl}\Delta M_{n} & =8071.43 & \mathrm{keV}, \text { mass excess of the neutron } \\ \Delta M_{H} & =7289.03 & \mathrm{keV}, \text { mass excess of hydrogen } \\ \alpha & =15971.3 & \mathrm{keV}, \text { volume-energy coefficient } \\ \beta & =30047 & \mathrm{keV}, \text { first symmetry-energy coefficient } \\ \gamma & =20806 & \mathrm{keV}, \text { surface-energy coefficient } \\ \eta & =45350 & \mathrm{keV}, \text { second symmetry-energy coefficient } \\ r_{0} & =1.16552 & \mathrm{fm}, \text { charge-radius constant } \\ \eta_{1} & =0 & \mathrm{keV}, \text { symmetry-energy parameter } \\ \eta_{2} & =\eta_{3}=-108.0 \mathrm{keV}, \text { pairing-energy parameters } \\ \eta_{4} & =\eta_{5}=0 & \mathrm{keV}, \text { pairing-energy parameters }\end{array}$

The functions $H_{1}(N)$ and $H_{2}(Z)$ are given in the table. The funclions $H_{\text {nucl }}(k) \equiv H_{1}(k)$ and $H_{\text {Coul }}(k) \equiv H_{2}(k)$ $-H_{1}(k)$ are displayed in Fig. 3. The standard deviation for the differences between calculated and experimental mass-excess values ( $N \geq 20$ and $Z \geq 20$ ) is $\sigma_{m}=289 \mathrm{kcV}$, the standard deviation for the differences between calculated and experimental Coulomb displacement energies is $\sigma_{c}=432 \mathrm{keV}$. About 5000 predicted mass values for nuclei with $A \geq 65$ are included in the tabulation.

It should be pointed out that multiparameter mass equations like the present one must be considered with some caution. It has been shown ${ }^{2}$ that $\chi^{2}$ per degree of freedom (which characterizes the goodness of fit) over the domain of measured mass values generally decreases inversely with the number of parameters. However, very little can be inferred from a small value of $\sigma_{m}$ alone about the expected reliability outside and particularly far away from the region of known masses. It is for this reason that other criteria ${ }^{2}$ including consistency test ${ }^{3}$ are important.

\section{References}

1. J. Jänecke and H. Behrens, Phys. Rev. C 9, 1276 (1974); Z. Phys. 256, 236 (1972); J. Jänecke, Phys. Rev. C 6, 467 (1972)

2. J. Jänecke and B. P. Eynon, Nucl. Phys. A 243, 326 (1975)

3. J. Jänecke and B. P. Eynon, in Proceedings of the Fifth International Conference on Atomic Masses and Fundamental Constants, Paris, France, 1975, edited by J. H. Sanders and A. H. Wapstra (Plenum Press, London-New York, 1975)

4. A. de-Shalit, Phys. Rev. 105, 1528 (1957)

5. G. T. Garvey et al., Rev. Mod. Phys. 41, S1 (1969)

6. A. H. Wapstra and N. B. Gove, Nuclear Data TABLES 9, 265 (1971)

7. N. Zeldes, A. Grill, and A. Simievic, K. Dan. Vidensk. Selsk. Mat.-Fys. Skr. 3, No. 5 (1967)

8. S. Liran and N. Zeldes, in International Conference on Nuclear Physics, Munich, 1973, edited by J. de Boer and H. J. Mang (North-Holland Publishing Co., Amsterdam, American-Elsevier, New York) p. 322; S. Liran, Ph.D. thesis, Hebrew University of Jerusalem (1973)

9. W. D. Myers and Swiatecki, Ann. Phys. (N.Y.) 55, 395 (1969); W. D. Myers, Nucl. Phys. A 145, 387 (1970); ibid. A 204, 465 (1973)

10. J. W. Truran, A. G. W. Cameron, and E. Hilf, in "International Conference on Properties of Nuclei Far from the Region of Beta-Stability, Leysin 1970," CERN Report No. 70-30 (Geneva) Vol. 1, p. 275

11. P. A. Seeger, in "International Conference on Properties of Nuclei Far from the Region of Beta-Stability, Leysin 1970," CERN Report No. 70-30 (Geneva) Vol. 1, p. 217

12. A. H. Wapstra and K. Bos, Atomic Data AND Nuclear Data Tables, this issue and private communication 
TABLE. Functions $H_{1}(N)$ and $H_{2}(Z)$ in $\mathrm{keV}$

\begin{tabular}{|c|c|c|c|c|c|c|c|c|c|c|}
\hline$H_{1}(l$ & & & & & & & & & & \\
\hline 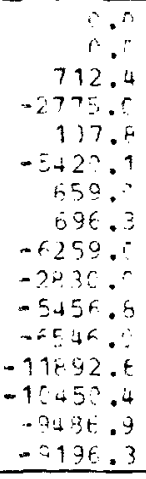 & 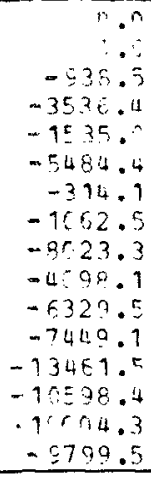 & $\begin{array}{r}7 . \\
250.7 \\
-1915.4 \\
-274.5 \\
-3956.1 \\
1193.6 \\
-283 . \\
-6196.7 \\
-3507 . \\
-5828.7 \\
-6899.7 \\
-13482 . \\
-9776.4 \\
-9486.0 \\
-9207.1 \\
\end{array}$ & $\begin{array}{r}0.9 \\
0.0 \\
-1876.1 \\
-2898.0 \\
-2000.6 \\
-4268 . ? \\
193.7 \\
-2057.2 \\
-6213.4 \\
-4597.8 \\
-6535.2 \\
-8194.4 \\
-14981.5 \\
-9911.3 \\
-10724.3 \\
-9254.8 \\
\end{array}$ & 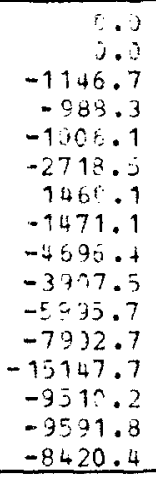 & $\begin{array}{r}0 . \\
-30 \\
-32.6 .0 \\
-2127.0 \\
-2958.9 \\
-3196.7 \\
419.6 \\
-3363.4 \\
-498.7 \\
-5121.0 \\
-6772.7 \\
-9447.4 \\
-15928.4 \\
-3654.5 \\
-9686.0 \\
-8734.8 \\
\end{array}$ & $\begin{array}{r}0.0 \\
\vdots .0 \\
-2681.7 \\
-383.7 \\
-2353.4 \\
-1341.0 \\
1709.1 \\
-2857.3 \\
-3468.7 \\
-4438.6 \\
-6279.4 \\
-9127.6 \\
-14113.8 \\
-9136.5 \\
-9377.5 \\
-9.5 \\
\end{array}$ & $\begin{array}{r}0.0 \\
-5041.3 \\
-1670.3 \\
-4658.3 \\
-1751.3 \\
392.0 \\
-4711.3 \\
-3845.2 \\
-5525.9 \\
-7297.3 \\
-10482.3 \\
-13648.9 \\
-9777.7 \\
-9803.4 \\
0.0\end{array}$ & $\begin{array}{r}0.0 \\
6.0 \\
-3736.1 \\
17.9 \\
-4484.3 \\
-129.7 \\
1353.0 \\
-4493.0 \\
-2456.1 \\
-4988.3 \\
-6560.4 \\
-10430.2 \\
-12015.9 \\
-9013.3 \\
-9352.4 \\
0.0 \\
\end{array}$ & $\begin{array}{r}0.0 \\
-1441.2 \\
-4389.6 \\
-1378.7 \\
-5924.9 \\
-968.3 \\
-146.7 \\
-6351.0 \\
-3626.9 \\
-5904.5 \\
-7130.5 \\
-11887.8 \\
-11973.7 \\
-9756.8 \\
-9837.4 \\
3.0 \\
\end{array}$ & $\begin{array}{l}10 \\
20 \\
30 \\
40 \\
50 \\
60 \\
70 \\
80 \\
90 \\
100 \\
110 \\
120 \\
130 \\
140 \\
150 \\
160 \\
\end{array}$ \\
\hline \multicolumn{11}{|l|}{$H_{2}(Z)$} \\
\hline $\begin{array}{r}0.0 \\
0.0 \\
751.5 \\
-19.1 \\
2151.7 \\
-1771.3 \\
2960.1 \\
5.38 . ? \\
3192.7 \\
8723.3 \\
\end{array}$ & $\begin{array}{r}0.0 \\
0.0 \\
-532.7 \\
-238.1 \\
75 . .5 \\
-2223.8 \\
2097.7 \\
4475.1 \\
2.89 .6 \\
7511.5\end{array}$ & $\begin{array}{r}0.0 \\
13.0 \\
735.5 \\
1615.1 \\
1813.3 \\
-42.00 \\
4168.5 \\
5285.5 \\
3955.7 \\
7982.3 \\
\end{array}$ & $\begin{array}{r}0.0 \\
0.0 \\
-852.5 \\
542.7 \\
-7.3 \\
-1096.8 \\
3139.9 \\
4389.9 \\
4372.6 \\
6865.4 \\
\end{array}$ & $\begin{array}{r}0.0 \\
0.0 \\
83.3 \\
2186.5 \\
320.3 \\
734.0 \\
4232.5 \\
5110.8 \\
5247.5 \\
7390.6 \\
\end{array}$ & $\begin{array}{r}0.0 \\
0.0 \\
-1625.3 \\
943.3 \\
-1633.9 \\
176.2 \\
3280.8 \\
4330.5 \\
6323.1 \\
6016.0 \\
\end{array}$ & $\begin{array}{r}0.0 \\
0.0 \\
-564.5 \\
2299.7 \\
-269.6 \\
2125.3 \\
4376.3 \\
5325.4 \\
7899.5 \\
6286.2 \\
\end{array}$ & $\begin{array}{r}0.0 \\
0.0 \\
-2485.9 \\
893.2 \\
-2174.3 \\
1657.3 \\
3633.9 \\
4079.7 \\
7711.4 \\
5210.3 \\
\end{array}$ & $\begin{array}{r}0.0 \\
0.0 \\
-788.7 \\
1967.4 \\
-1445.4 \\
3264.4 \\
4633.5 \\
4416.8 \\
8786.8 \\
5460.8 \\
\end{array}$ & $\begin{array}{r}0.0 \\
-1441.2 \\
-1203.5 \\
657.2 \\
-3571.0 \\
2515.1 \\
3969.8 \\
3024.8 \\
8198.0 \\
4502.9 \\
\end{array}$ & $\begin{array}{l}10 \\
20 \\
30 \\
40 \\
50 \\
60 \\
70 \\
80 \\
90 \\
100 \\
\end{array}$ \\
\hline
\end{tabular}

The first line gives $H_{1}(1), H_{1}(2), \ldots, H_{1}(10)$; the second line gives $H_{1}(11), H_{1}(12), \ldots, H_{1}(20)$, etc. 